\title{
Gain of Virulence Caused by Insertion of a Pot3 Transposon in a Magnaporthe grisea Avirulence Gene
}

\author{
Seogchan Kang, Marc Henri Lebrun, Leonard Farrall, and Barbara Valent \\ DuPont Central Research and Development, Experimental Station, Wilmington, DE 19880-0402, U.S.A. \\ Accepted 10 January 2001.
}

\begin{abstract}
The avirulence gene AVR-Pita in Magnaporthe grisea prevents the fungus from infecting rice cultivars carrying the disease resistance gene $\mathrm{Pi}$-ta. Insertion of Pot3 transposon into the promoter of $A V R$-Pita caused the gain of virulence toward Yashiro-mochi, a rice cultivar containing Pi-ta, which demonstrated the ability of Pot 3 to move within the $M$. grisea genome. The appearance of Pot3 in $M$. grisea seems to predate the diversification of various hostspecific forms of the fungus.
\end{abstract}

Additional keywords: Pyricularia grisea; race variation.

Rice blast caused by Magnaporthe grisea (Hebert) Barr. (anamorph Pyricularia grisea Sacc.) is one of the most economically devastating crop diseases worldwide (Zeigler et al. 1994). Management of rice blast through the breeding of blast-resistant cultivars has had only limited success as a result of the frequent breakdown of resistance under field conditions (Zeigler et al. 1994). The frequent appearance of new races or pathotypes of the fungus has been proposed as the principal mechanism involved in the loss of resistance. Because the compatibility of interaction between $M$. grisea and rice follows the gene-for-gene model (Bryan et al. 2000; Jia et al. 2000; Orbach et al. 2000; Zeigler et al. 1994), it has been hypothesized that loss-of-function mutations of the avirulence gene could lead to the generation of new races. Cloning avirulence genes from $M$. grisea showed that such mutations frequently occur in some isolates (Orbach et al. 2000; Sweigard et al. 1995). Analysis of the spontaneous mutants of $M$. grisea, which gained virulence on weeping lovegrass, showed that $P W L 2$, an avirulence gene preventing the fungus from infecting this plant, had been deleted in these mutants (Sweigard et al. 1995). The AVR-Pita gene, which corresponds in a gene-for-gene fashion to the disease resistance

Corresponding author: S. Kang; Current address: Department of Plant Pathology, Pennsylvania State University, University Park, 16802, U.S.A.; Telephone: +1-814-863-3846; Fax: +1-814-863-7217; E-mail: sxk55@psu.edu

Current address of M. H. Lebrun: UMR 1932 CNRS-Aventis Crop Science, 14 rue P. Baizet, Lyon, France.

Current address of L. Farrall and B. Valent: DuPont Agricultural Products, P. O. Box 80402, Wilmington, DE 19880-0402, U.S.A. gene Pi-ta, was cloned and characterized (Orbach et al. 2000).

The AVR-Pita gene is located entirely within a $1.5-\mathrm{kb}$ telomeric fragment at one end of chromosome 3, and it encodes a putative metalloprotease (Orbach et al. 2000). Spontaneous mutants of $M$. grisea that had gained virulence specifically toward Yashiro-mochi, a rice cultivar carrying $P i$-ta, exhibited diverse mutations in AVR-Pita. Three of the 11 mutants characterized by Orbach et al. (2000) contained point mutations in the AVR-Pita coding sequence and seven contained deletions of AVR-Pita gene sequences. The remaining mutant, CP1632, contained a $1.8-\mathrm{kb}$ insertion located between the $S a c \mathrm{I}$ and EcoRI sites of AVR-Pita (Fig. 1). In this report, we describe the cloning and characterization of this insertion.

Southern analysis of CP1632 with several restriction fragments between the $S a c \mathrm{I}$ and $E c o$ RI sites as probes showed that the insertion occurred between the ApaI and the HindIII site nearest to the open reading frame (ORF) of AVR-Pita (Fig. 1). Genomic DNA of CP1632 was amplified by polymerase chain reaction (PCR) with primers F3 and F4 flanking the HindIII and $A p a \mathrm{I}$ sites, respectively (Fig. 1). A 2-kb fragment amplified by PCR hybridized to the 500-bp probe A, which spans the insertion site (Fig. 1), confirming the successful amplification of the desired product. The amplified fragment hybridized to a large number of bands in CP1632 and other rice pathogens of $M$. grisea, suggesting that the insertion corresponds to a repetitive DNA element. The fragment was treated with T4 DNA polymerase in the presence of deoxynucleoside triphosphates to make its ends blunt, then digested with HindIII and subsequently cloned into a SmaI-HindIII-digested pBluescriptSK (Stratagene, La Jolla, CA, U.S.A.).

Sequence of the insertion in CP1632 showed that it belongs to the transposon family Pot3. The insertion site of Pot3 in AVR-Pita was located 304 bp upstream from the initiation codon of the AVR-Pita ORF. Orbach et al. (2000) used complementation analysis to show that subclones containing at least $477 \mathrm{bp}$ of the $5^{\prime}$ sequence were active in conferring avirulence to Yashiro-mochi when transformed into a virulent fungal strain, but neither the telomeric HindIII fragment with $219 \mathrm{bp}$ of the $5^{\prime}$ sequence nor the telomeric ApaI fragment with $389 \mathrm{bp}$ of the $5^{\prime}$ sequence was active in conferring avirulence (Fig. 1). Therefore, sequences essential for the gene function were located upstream from the insertion site. To rule out the possibility that an additional mutation in AVR-Pita caused the loss-of-gene function in CP1632, the 1,273-bp 
region of AVR-Pita between the telomeric repeat and the HindIII site (Fig. 1) was amplified by PCR and sequenced. The resulting sequence was identical to the wild-type allele. Thus, the insertion of Pot 3 between the ApaI and HindIII sites is the only change detected relative to the functional AVR-Pita gene, and this insertion physically separated critical promoter sequences from the rest of the gene by $1.8 \mathrm{~kb}$, causing the disruption of the avirulence gene expression.

Pot3 was identified originally as part of MGR586 (Farman et al. 1996a; Hamer et al. 1989), a repetitive DNA probe used extensively for population analysis of $M$. grisea (Levy et al. 1991; Zeigler et al. 1994). Pot3 contains two inverted terminal repeats (ITR) and one ORF exhibiting significant homology to transposases encoded by TC1/Mariner transposons (Farman et al. 1996a). The copy of Pot3 inserted in AVR-Pita (GenBank accession no. AF333034) was nearly identical to the previously sequenced copy (Farman et al. 1996a), with the following differences. As a result of one additional base pair near the $3^{\prime}$ end of the ORF in the former, it (556 amino acids) is slightly larger than the latter (554 amino acids), and their carboxy termini (approximately 50 amino acids) are completely different. Transposition of Pot3 into AVR-Pita duplicated the target site (5'-TA-3'), which has been observed in other families of TC1/Mariner-like transposons, including Pot 2 in $M$. grisea (Kachroo et al. 1994) and Fot1 in Fusarium oxysporum (Daboussi et al. 1992). To investigate whether any sequence preference exists for target sites of Pot3, eight independent cosmid clones carrying a single copy of Pot3 were isolated from a library of 4375-R-6 (the wild-type strain of CP1632). We determined the sequence of their ITR and flanking genomic DNA with primers P3 and P4 (Fig. 1). The ITR of six of these Pot3 elements was identical to that of the copy inserted in AVR-Pita. The remaining two copies differed from the rest by either a point mutation in its $5^{\prime}$ ITR or the absence of one copy of the direct repeat in its $3^{\prime}$ ITR (not shown). Sequences flanking these elements did not exhibit significant similarity, except for the duplicated 2-bp target (Fig. 2).

On the basis of the nearly ubiquitous presence of sequences homologous to MGR586 among isolates from diverse hosts, it was hypothesized that the appearance of Pot3 in the $M$. grisea genome may predate the diversification of various hostspecific forms of the fungus (Shull and Hamer 1994). To test this hypothesis rigorously, we surveyed 50 isolates from more than 20 host species other than rice for sequences homologous to Pot3 (Fig. 3). Southern analysis of the EcoRI-digested genomic DNAs of these isolates was carried out with probe B
Locus
Target Sequence
AVR-Pita
Cosmid 1
Cosmid 2
Cosmid 3
Cosmid 4
Cosmid 5
Cosmid 6
Cosmid 7
Cosmid 8
5'-TTGACATTAAGCATATATTGTCCGGCGG-3'

Fig. 2. Comparison of the genomic sequences flanking nine copies of Pot3. The sequence duplicated by insertion of Pot $3\left(5^{\prime}-\mathrm{TA}-3^{\prime}\right)$ is in bold.

$1 \mathrm{~kb}$
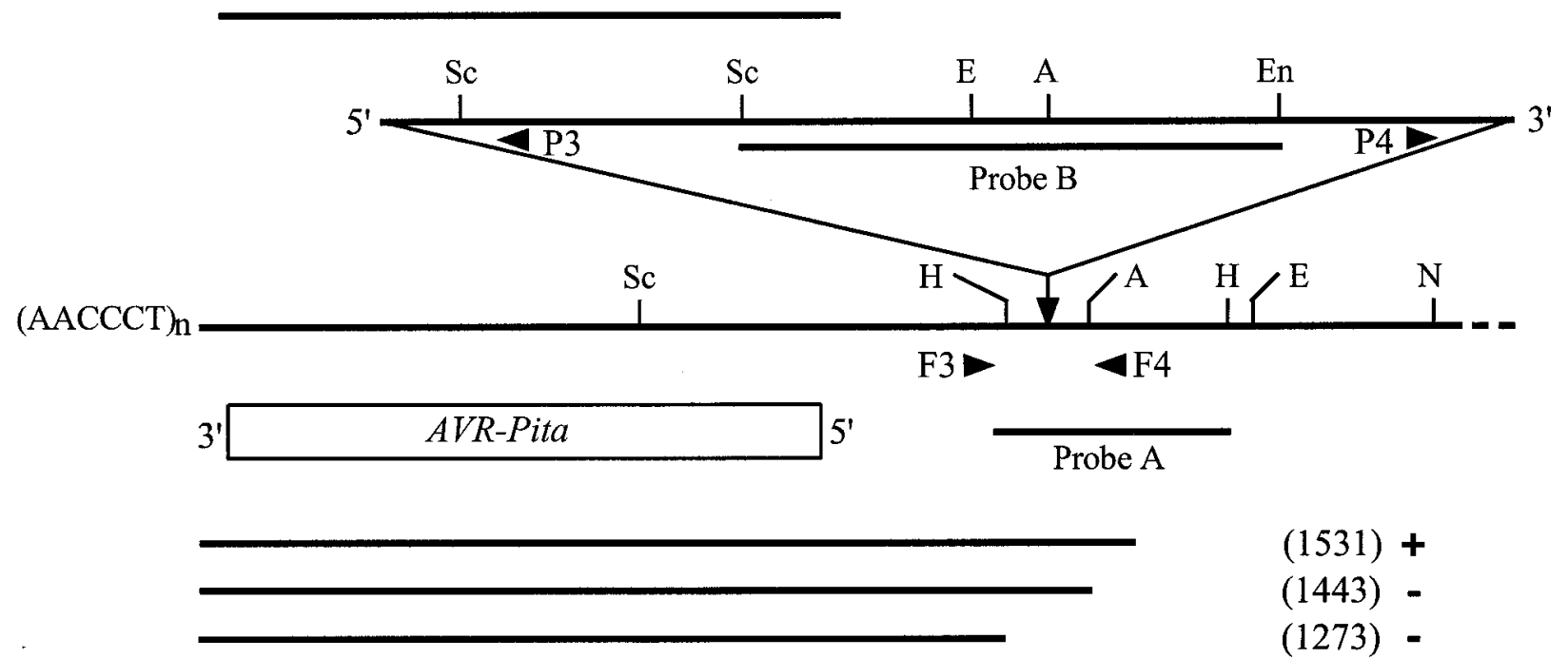

Fig. 1. Restriction map of the AVR-Pita locus in CP1632. Restriction sites for ApaI (A), EcoRI (E), EcoNI (En), HindIII (H), NcoI, and SacI (Sc) are marked. The telomeric repeat sequence $\left(5^{\prime}\right.$-AACCCT- $\left.3^{\prime}\right)$ was denoted by (AACCCT)n. The open reading frame of $A V R$-Pita is shown below the restriction map. Two arrowheads below the restriction map of the AVR-Pita locus indicate the primers used to amplify the inserted sequence in CP1632: $\mathrm{F} 3$ ( $5^{\prime}$ TTATTATTTCAGCTTGACT-3') and F4 (5'-CATGAATTATGTAGTTATT-3'). Probe A was used to confirm the successful amplification of the inserted DNA. The SacI-EcoNI fragment underneath the restriction map of Pot3, labeled probe B, was used to survey the distribution pattern of Pot3 (Fig. 3 ). Location of the two primers for determining the sequences flanking Pot3, P3 (5'-GCTTCGCAATTTGATGATTC-3' and P4 (5'-GATGTACATATATCCG ATTG-3'), is shown underneath the restriction map of Pot3. The location and sizes (in base pairs) of three subclones of the $A V R-P i t a$ locus, which were used to determine the sequences required for the gene function (Orbach et al. 2000), are indicated below the restriction map. A 1,531-bp telomere end fragment was active (+) in conferring avirulence toward cultivar Yashiro-Mochi, but the 1,443-bp ApaI fragment and the 1,273-bp HindIII fragment were inactive (-) (Orbach et al. 2000). 
(Fig. 1) under high-stringency conditions. Because there is an EcoRI site in the middle of probe B, the copy number of Pot3 is likely to be about one-half of the number of bands observed. All the isolates except two, G-227 from ginger and G-229 from Cyperus spp., contained one to 50 bands that hybridized to the probe. This result confirms that Pot3 is nearly ubiqui- tous in $M$. grisea, thus supporting the hypothesis of Shull and Hamer (1994).

An isolate from barley, G-197, and three isolates from Pennisetum spp., G-222, G-223, and G-224, had a high copy number of Pot3, comparable to that of isolates from rice. Isolate G-197 has a mitochondrial DNA type that is identical to
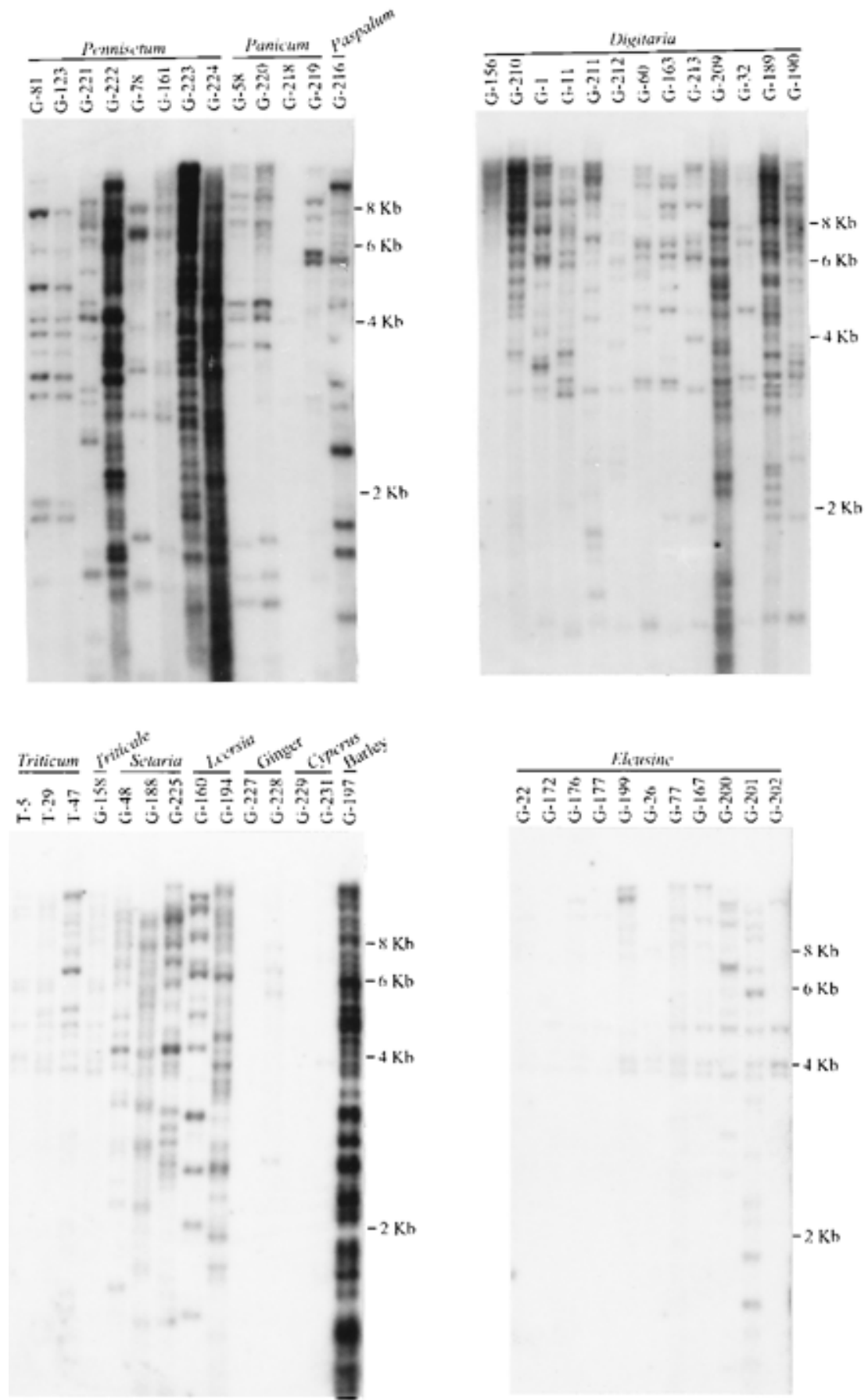

Fig. 3. Distribution patterns of Pot3 among isolates of Magnaporthe grisea from diverse host species other than rice. Genomic DNA of these isolates was digested with EcoRI, separated by electrophoresis on a $0.7 \%$ agarose gel, and blotted onto a nylon membrane (Hybond-N, Amersham Pharmacia, Buckinghamshire, U.K.). Hybridization of the membranes with labeled probe B (Fig. 1) and subsequent washings were performed in highly stringent conditions: $65^{\circ} \mathrm{C}$ in $6 \times \mathrm{SSC}(1 \times \mathrm{SSC}$ is $0.15 \mathrm{M} \mathrm{NaCl}$ plus $0.015 \mathrm{M}$ sodium citrate), $2 \times$ Denhardt's reagent, and $0.1 \%$ sodium dodecyl sulfate (SDS) for hybridization, followed by a 30-min. wash at $65^{\circ} \mathrm{C}$ in $1 \times$ SSC and $0.1 \%$ SDS and an additional 30-min. wash in $0.2 \times$ SSC and $0.1 \%$ SDS. The genus (or common name) of the host plant from which individual strains were isolated is shown on the top of each figure. Detailed information on the origin of these strains is in Kang et al. (1995). 
isolates from rice and infects rice and barley (B. Valent, unpublished), suggesting that G-197 is highly related to rice pathogens. Isolates from Pennisetum spp., however, are genetically distinct from rice pathogens (Shull and Hamer 1994) and are not pathogenic on rice. The high copy number of Pot3 in such isolates has resulted from amplification events independent of the amplification observed in the genome of isolates pathogenic on rice. A survey of isolates from Oryza spp. showed that two strains (O-312 and O-315) isolated from two wild rice species, Oryza glaberrima and Oryza longistaminata, respectively, contained less than 10 copies of Pot3 (S. Kang, unpublished results). These results indicate that the presence of a high copy number of Pot 3 is not necessarily a unique characteristic of strains infecting rice.

It has been suggested that genetic changes caused by transposons may be one of the mechanisms responsible for race variation (Shull and Hamer 1994; Talbot et al. 1993; Valent and Chumley 1991). Recombination between members of the same family may cause such mutations as deletion, inversion, duplication, and translocation, depending on the relative orientation and position of the members that are recombined. In addition, some transposons are mobile and, as a result of insertion or excision, can cause mutations. Different types of transposons have been identified in M. grisea (Dobinson et al. 1993; Farman et al. 1996a; Farman et al. 1996b; Hamer et al. 1989; Kachroo et al. 1994; Kachroo et al. 1995; Sone et al. 1993; Valent and Chumley 1991). These include retrotransposons that transpose through an RNA intermediate and DNA transposons that directly transpose by a cut-and-paste mechanism. Two of the retrotransposons, MAGGY and MGL, have been shown to transpose (Nishimura et al. 2000; Shull and Hamer 1996). Our work demonstrates that the DNA transposon Pot3 can transpose and provides evidence that its transposition can change the virulence spectrum of $M$. grisea.

\section{ACKNOWLEDGMENTS}

We are grateful to J.-R. Xu at Purdue University for providing cosmid clones containing Pot3.

\section{LITERATURE CITED}

Bryan, G. T., Wu, K., Farrall, L., Jia, Y., Hershey, H. P., McAdams, S. A., Faulk, K. N., Donaldson, G. K., Tarchini, R., and Valent, B. 2000. A single amino acid difference distinguishes resistant and susceptible alleles of the rice blast resistance gene Pi-ta. Plant Cell 12:2033-2046.

Daboussi, M. J., Langin, T., and Brygoo, Y. 1992. Fot1, a new family of fungal transposable elements. Mol. Gen. Genet. 232:12-16.

Dobinson, K. F., Harris, R. E., and Hamer, J. E. 1993. Grasshopper, a long terminal repeat (LTR) retroelement in the phytopathogenic fungus Magnaporthe grisea. Mol. Plant-Microbe Interact. 6:114-126.

Farman, M. L., Taura, S., and Leong, S. A. 1996a. The Magnaporthe grisea DNA fingerprinting probe MGR586 contains the $3^{\prime}$ end of an inverted repeat transposon. Mol. Gen. Genet. 251:675-681.

Farman, M. L., Tosa, Y., Nitta, N., and Leong, S. A. 1996b. MAGGY, a retrotransposon in the genome of the rice blast fungus Magnaporthe grisea. Mol. Gen. Genet. 251:665-674.

Hamer, J. E., Farrall, L., Orbach, M. J., Valent, B., and Chumley, F. G. 1989. Host species-specific conservation of a family of repeated DNA sequences in the genome of a fungal plant pathogen. Proc. Natl. Acad. Sci. USA 86:9981-9985.

Jia, Y., McAdams, S. A., Bryan, G. T., Hershey, H. P., and Valent, B. 2000. Direct interaction of resistance gene and avirulence gene products confers rice blast resistance. EMBO J. 19:4004-4014.

Kachroo, P. K., Chattoo, B. B., and Leong, S. A. 1994. Pot2, an inverted repeat transposon from Magnaporthe grisea. Mol. Gen. Genet. 245:339-348.

Kachroo, P., Leong, S. A., and Chattoo, B. B. 1995. Mg-SINE: A short interspersed nuclear element from the rice blast fungus, Magnaporthe grisea. Proc. Natl. Acad. Sci. USA 92:11125-11129.

Kang, S., Sweigard, J. A., and Valent, B. 1995. The $P W L$ host specificity gene family in the blast fungus Magnaporthe grisea. Mol. PlantMicrobe Interact. 8:939-948.

Levy, M., Romao, J., Marchetti, M. A., and Hamer, J. E. 1991. DNA fingerprinting with a dispersed repeated sequence resolves pathotype diversity in the rice blast fungus. Plant Cell 3:95-102.

Nishimura, M., Hayashi, N., Jwa, N.-S., Lau, G. W., Hamer, J. E., and Hasebe, A. 2000. Insertion of the LINE retrotransposon MGL causes a conidiophore pattern mutation in Magnaporthe grisea. Mol. PlantMicrobe Interact. 13:892-894.

Orbach, M. J., Farrall, L., Sweigard, J. A., Chumley, F. G., and Valent, B. 2000. A telomeric avirulence gene determines efficacy for rice blast resistance gene Pi-ta. Plant Cell 12:2019-2032.

Shull, V., and Hamer, J. E. 1994. Genomic structure and variability in Pyricularia grisea. Pages 65-86 in: Rice Blast Disease. R. S. Zeigler, P. S. Teng, and S. A. Leong, eds. CAB International, Wallingford, England.

Shull, V., and Hamer, J. E. 1996. Rearrangement at a DNA-fingerprint locus in the rice blast fungus. Curr. Genet. 30:263-271.

Sone, T., Suto, M., and Tomita, F. 1993. Host species-specific repetitive DNA sequence in the genome of Magnaporthe grisea, the rice blast fungus. Biosci. Biotech. Biochem. 57:1228-1230.

Sweigard, J. A., Carroll, A. M., Kang, S., Farrall, L., Chumley, F. G., and Valent, B. 1995. Identification, cloning, characterization of PWL2, a gene for host-species specificity in the rice blast fungus. Plant Cell 7:1221-1233.

Talbot, N. J., Salch, Y. P., Ma, M., and Hamer, J. E. 1993. Karyotypic variation within clonal lineages of the rice blast fungus, Magnaporthe grisea. Appl. Environ. Microbiol. 59:585-593.

Valent, B., and Chumley, F. G. 1991. Molecular genetic analysis of the rice blast fungus, Magnaporthe grisea. Annu. Rev. Phytopathol. 29:443-467.

Zeigler, R. S., Leong, S. A., and Teng, P. S. 1994. Rice Blast Disease. $\mathrm{CAB}$ International, Wallingford, England. 